\title{
Safety and immunogenicity of a self-amplifying RNA vaccine against COVID-19: COVAC1, a phase I, dose- ranging trial
}

Katrina M. Pollock, ${ }^{a, b}$ Hannah M. Cheeseman, ${ }^{a}$ Alexander J. Szubert, ${ }^{c}$ Vincenzo Libri, ${ }^{d}$ Marta Boffito, ${ }^{a, e}$ David Owen, ${ }^{b}$ Henry Bern, Jessica O'Hara, ${ }^{a}$ Leon R. McFarlane, ${ }^{a}$ Nana-Marie Lemm, ${ }^{a}$ Paul F. McKay, ${ }^{a}$ Tommy Rampling, ${ }^{d}$ Yee Ting N. Yim, ${ }^{d}$ Ana Milinkovic, ${ }^{e}$ Cherry Kingsley, ${ }^{a}$ Tom Cole, ${ }^{b}$ Susanne Fagerbrink, ${ }^{b}$ Marites Aban, ${ }^{b}$ Maniola Tanaka, ${ }^{b}$ Savviz Mehdipour, ${ }^{b}$ Alexander Robbins, ${ }^{b}$ William Budd, ${ }^{b}$ Saul N. Faust, ${ }^{f}$ Hana Hassanin, ${ }^{g}$ Catherine A. Cosgrove, ${ }^{h}$ Alan Winston, ${ }^{a}$ Sarah Fidler, ${ }^{a}$ David T. Dunn, ${ }^{c}$

Sheena McCormack, ${ }^{c}$ and Robin J. Shattock ${ }^{a *}$, on behalf of the COVAC1 study Group ${ }^{\dagger}$

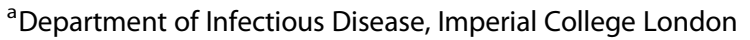

${ }^{b}$ NIHR Imperial Clinical Research Facility and NIHR Imperial Biomedical Research Centre, London, UK

${ }^{\mathrm{C}}$ MRC Clinical Trials Unit at UCL, London, UK

${ }^{\mathrm{d}}$ NIHR UCLH Clinical Research Facility and NIHR UCLH Biomedical Research Centre, London, UK

${ }^{e}$ Chelsea \& Westminster Hospital, London

${ }^{f}$ NIHR Southampton Clinical Research Facility and Biomedical Research Centre, University Hospital Southampton NHS

Foundation Trust, Southampton, UK; Faculty of Medicine and Institute for Life Sciences, University of Southampton,

Southampton, UK

${ }^{9}$ Surrey Clinical Research Facility, Faculty of Health and Medical Sciences, University of Surrey, Guildford, UK

${ }^{\mathrm{h}}$ Centre for Infection, St George's, University of London, London, United Kingdom

\section{Summary}

Background Lipid nanoparticle (LNP) encapsulated self-amplifying RNA (saRNA) is a novel technology formulated as a low dose vaccine against COVID-I9.

Methods A phase I first-in-human dose-ranging trial of a saRNA COVID-I9 vaccine candidate LNP-nCoVsaRNA, was conducted at Imperial Clinical Research Facility, and participating centres in London, UK, between $19^{\text {th }}$ June to $28^{\text {th }}$ October 2020. Participants received two intramuscular (IM) injections of LNP-nCoVsaRNA at six different dose levels, 0.I-I0.0 $\mathrm{g}$, given four weeks apart. An open-label dose escalation was followed by a dose evaluation. Solicited adverse events (AEs) were collected for one week from enrolment, with follow-up at regular intervals (I-8 weeks). The binding and neutralisation capacity of anti-SARS-CoV-2 antibody raised in participant sera was measured by means of an anti-Spike (S) IgG ELISA, immunoblot, SARS-CoV-2 pseudoneutralisation and wild type neutralisation assays. (The trial is registered: ISRCTNi7072692, EudraCT 2020-001646-20).

Findings 192 healthy individuals with no history or serological evidence of COVID-19, aged I8-45 years were enrolled. The vaccine was well tolerated with no serious adverse events related to vaccination. Seroconversion at week six whether measured by ELISA or immunoblot was related to dose (both p<0.00I), ranging from 8\% (3/39; $0 . \mathrm{I} \mu \mathrm{g})$ to $6 \mathrm{I} \%(\mathrm{I} 4 / 23 ; 10.0 \mu \mathrm{g})$ in ELISA and $46 \%(\mathrm{I} 8 / 39 ; 0.3 \mu \mathrm{g})$ to $87 \%(20 / 23 ; 5.0 \mu \mathrm{g}$ and $\mathrm{I0.0} \mu \mathrm{g})$ in a post-hoc immunoblot assay. Geometric mean (GM) anti-S IgG concentrations ranged from 74 (95\% CI, 45 -II9) at $0 . \mathrm{I} \mu \mathrm{g}$ to $1023(468-2236) \mathrm{ng} / \mathrm{mL}$ at $5.0 \mu \mathrm{g}(\mathrm{p}<0.00 \mathrm{I})$ and was not higher at $10.0 \mu \mathrm{g}$. Neutralisation of SARS-CoV-2 by participant sera was measurable in $15 \%(6 / 39 ; 0.1 \mu \mathrm{g})$ to $48 \%(\mathrm{II} / 23 ; 5.0 \mu \mathrm{g})$ depending on dose level received.

Interpretation Encapsulated saRNA is safe for clinical development, is immunogenic at low dose levels but failed to induce $100 \%$ seroconversion. Modifications to optimise humoral responses are required to realise its potential as an effective vaccine against SARS-CoV-2.

Funding This study was co-funded by grants and gifts from the Medical Research Council UKRI (MC_PC_I9076), and the National Institute Health Research/Vaccine Task Force, Partners of Citadel and Citadel Securities, Sir
EClinicalMedicine 2022;44: 101262 Published online xxx https://doi.org/10.1016/j. eclinm.2021.101262

Abbreviations: AEs, adverse events; GOI, gene of Interest; LNP, lipid nanoparticle; NSP, non-structural protein; saRNA, self-amplifying RNA; VEEV, Venezuelan equine encephalitis virus

*Corresponding author.

E-mail address: r.shattock@imperial.ac.uk (R.J. Shattock).

$\dagger$ Listed in the Supplementary. 
Joseph Hotung Charitable Settlement, Jon Moulton Charity Trust, Pierre Andurand, Restore the Earth.

Copyright (C) 202I The Authors. Published by Elsevier Ltd. This is an open access article under the CC BY-NC-ND license (http://creativecommons.org/licenses/by-nc-nd/4.०/)

\section{Research in context}

\section{Evidence before this study}

Lipid nanoparticle (LNP) encapsulated self-amplifying (sa) RNA is a novel technology for use in vaccines and therapeutics. Alphavirus replicons have shown promise in preclinical models against multiple targets. We searched PubMed from June 22, 2012 to Oct 9, 2021, with the search terms "self-amplifying RNA" AND "clinical trial", and no clinical studies assessing the safety and immunogenicity of LNP encapsulated saRNA had been published.

\section{Added value of this study}

Here we observed that clinical use of an saRNA vaccine (LNP-nCoVsaRNA) expressing the pre-fusion stabilised spike (S) glycoprotein of SARS-CoV-2 raised no shortterm safety concerns and was well tolerated, with fewest reactions at low doses. The vaccine was immunogenic but neither seroconversion rates, up to $61 \%$ (14/ $23 ; 10.0 \mu \mathrm{g})$, measured by ELISA and $87 \%(20 / 23 ; 5.0 \mu \mathrm{g}$ and $10.0 \mu \mathrm{g}$ ) measured by immunoblot, nor SARS-CoV-2 virus neutralisation rates reached $100 \%$ in any dose group.

\section{Implications of all the available evidence}

To our knowledge, this study is the first to provide safety and immunogenicity data on the use of encapsulated saRNA for vaccine development including pandemic COVID-19 vaccines. Human responses to SARSCoV-2 were significantly lower than those predicted by small animal models. Understanding species specific differences in innate regulation of saRNA expression may prove critical to improved vector design and development of better preclinical models.

\section{Introduction}

Lipid nanoparticle (LNP) encapsulated self-amplifying RNA (saRNA) is a novel technology with significant, and previously untested, potential for translation into human use in the development of novel drugs and vaccines. Vaccines against COVID-I9 require production technology that is highly scalable to meet global demand and readily modifiable to combat the emergence of new SARS-CoV-2 variants, particularly those with the potential to escape vaccine-induced immunity. COVID-I9 vaccines may also be needed that allow for easy and acceptable repeat administration to boost immune responses that wane after initial vaccination or recovery from natural infection. ${ }^{2}$ Vaccines developed using encapsulated saRNA have unique features, which include low dose administration and a readily modifiable antigenic domain making it possible to formulate vaccines rapidly.

Venezuelan equine encephalitis virus (VEEV) is an RNA virus which encodes a cytoplasmic replicon. Previous clinical experience of alphavirus replicons utilised virus like particles (VLP) derived from an attenuated VEEV strain. CMV and HIV vaccines based on this design induced modest neutralising and binding antibody responses respectively in phase I clinical trials, although anti-VLP immunity was also observed after immunisation. ${ }^{3,4}$

We developed a candidate LNP encapsulated saRNA COVID-I9 vaccine which demonstrated anti-SARSCoV-2 immunogenicity in small animals even at ultralow doses (0.0I $\mu \mathrm{g}) .^{5}$ The vaccine is II, 507 nucleotides in length and encodes the replicase composed of nonstructural proteins (NSPs I-4) from the VEEV as well as a pathogen-specific immunogen, the spike (S) glycoprotein from SARS-CoV-2. Manufacture of the vaccine is entirely synthetic and does not require any form of virus culture, a significant hurdle in scalability and fidelity to the wild-type virus strains for many licensed vaccines. ${ }^{6,7}$

The vaccine is injected intramuscularly and LNP encapsulation facilitates endosomal uptake and release into the cytoplasm of target cells in vivo. The VEEV replicase encodes non-structural proteins for self-amplification. ${ }^{8}$ The replicase, generated as a polyprotein (NSP I-3 $\& 4$ ), initially amplifies the complete saRNA sequence, but following a process of self-cleavage into individual components (NSP I,2,3\&4) rapidly switches to preferential amplification of the gene-of-interest (GOI) driven by the internal subgenomic promotor. ${ }^{8}$ No potentially infectious structural proteins are expressed, as these have been replaced by the vaccine GOI, a codon-optimised SARS-CoV-2 S glycoprotein. The full-length $\mathrm{S}$ glycoprotein is presented as a membrane tethered trimer and includes two proline amino acid substitutions (2P-S, K968P and V969P) that, when expressed, stabilise it in the prefusion conformation rendering it incapable of facilitating fusion. ${ }^{9}$

Expression kinetics of the vaccine in mice indicate protein expression to peak at seven days and resolve by 2I days, similar kinetics are observed when injected ex vivo into human skin explants. ${ }^{\text {IO,II }}$ Pre-clinical toxicology studies in rats indicated no observed adverse events up to $10.0 \mu \mathrm{g}$ (the maximum dose tested). Importantly the potential of this technology predicted from numerous preclinical studies has not previously been assessed 
in humans. We report the preliminary findings of a phase I dose ranging trial to assess the safety and immunogenicity of six dose levels (ranging from o.I $\mu \mathrm{g}$ to $10.0 \mu \mathrm{g}$ ) of the candidate saRNA COVID-I9 vaccine, LNP-nCoVsaRNA.

\section{Methods}

\section{Study design and participants}

We report the results up to 8 weeks of the dose escalation and evaluation components of a protocol that proceeds to an expanded safety cohort in preparation for an efficacy trial (see protocol version 8.0, Appendix I). Initially, three different doses (0.I $\mu \mathrm{g}, 0.3 \mu \mathrm{g}, \mathrm{I} .0 \mu \mathrm{g}$ ) were assessed in $\mathrm{I} 20$ participants enrolled at a single centre (NIHR Imperial Clinical Research Facility). Following review of safety and immune responses by the Trial Steering Committee, three higher doses $(2.5 \mu \mathrm{g}, 5.0 \mu \mathrm{g}$, Io. $0 \mu \mathrm{g}$ ) were assessed in 72 participants enrolled from three centres (NIHR Imperial Clinical Research Facility; Chelsea and Westminster Hospital NHS Foundation Trust; NIHR UCLH Clinical Research Facility, University College London Hospitals NHS Foundation Trust).

Healthy participants aged $\mathrm{I} 8-45$ years were recruited through local advertisements. Participants with no history of COVID-I9 were eligible to take part. All participants underwent a screening visit where a full medical history and examination was performed in addition to blood and urine tests. Participant sera were screened for antibodies against SARS-CoV-2 using the Abbott Architect nucleocapsid IgG assay (N IgG) and for the presence of blood borne viruses using a fourth generation HIV test and for IgG against Hepatitis C. Those with reactive responses in any of these tests were ineligible for the study. Full details of the eligibility criteria are described in Appendix 2.

Written informed consent was obtained from all participants, and the trial conducted in accordance with the principles of the Declaration of Helsinki and Good Clinical Practice. Participants were offered reimbursement for their time, inconvenience, and travel expenses of $£ 50$ per visit paid as a lump sum at the end of the participation. This study was approved in the UK by the Medicines and Healthcare products Regulatory Agency and the North East - York Research Ethics Committee (reference 20/SC/OI45) (ISRCTNI7072692, EudraCT 2020oor646-20).

\section{Randomisation and masking}

Both the lower and higher dose cohorts included an open-label dose escalation phase (I5 and I2 participants respectively). Subsequently, participants were allocated in a I:I:I ratio (centralised computer-generated list, block size 3) to the three doses either by individual randomisation (lower dose cohort) or based on the day when they were vaccinated (higher dose cohort).
Additional detail is provided in Appendix 2, Table I. Laboratory staff and participants (apart from the dose escalation phase) were blinded to the dose allocation.

\section{Procedures}

LNP-nCoVsaRNA is a self-amplifying ribonucleic acid (saRNA) vaccine, II,507 nucleotides in length, encapsulated within lipid nanoparticles (LNPs). It encodes two major components; the non-structural replicase proteins from VEEV epizootic Trinidad Donkey strain and the spike (S) glycoprotein of SARS-CoV-2 (GenBank accession number: QHD434I6.I) stabilised in the prefusion conformation by the insertion of two proline substitutions K986 and V987. 9 The saRNA is generated from a DNA template by in vitro transcription with co-transcriptional capping $\left(\mathrm{m} 7 \mathrm{G}\left(5^{\prime}\right) \operatorname{ppp}\left(5^{\prime}\right)\left(2^{\prime} \mathrm{OMeA}\right) \mathrm{pU}\right.$; TriLink). The RNA is formulated with lipids (ionisable lipid (proprietary to Acuitas)/phosphatidylcholine/cholesterol/PEG-lipid) to obtain the LNP-nCoVsaRNA drug product.

Conduct of the trial. The trial evaluated six dose levels of LNP-nCoVsaRNA as two intramuscular (IM) injections into the deltoid muscle of the non-dominant arm, given four weeks apart. The vaccine was formulated as a suspension for injection in multi-dose vials stored at $-70^{\circ} \mathrm{C}$. On the day of injection, it was thawed and diluted in phosphate buffered saline (PBS) to give a final volume of $0.5 \mathrm{~mL}$ for injection for dose levels from I.o $\mu \mathrm{g}$ to I0.० $\mu \mathrm{g}$. The o.I $\mu \mathrm{g}$ and $0.3 \mu \mathrm{g}$ doses were prepared to give a final volume for injection of $0.2 \mathrm{~mL}$ and $0.6 \mathrm{~mL}$ respectively. Participants were observed for up to one hour following each immunization.

Solicited adverse events (AEs) were self-reported by participants in electronic diary records captured the evening after injection and for six further evenings. Study staff checked the diary record approximately 48 hours later and at the day seven visit. All these events were considered related to vaccination. Causality was determined by the site investigators. Blood samples were collected at days 7, I4, 28, 35, 42 and 56 (haemoglobin, white cell count, platelets, lymphocytes, neutrophils, transaminases, total bilirubin, serum creatinine and non-fasting glucose). Grade was determined according to the FDA toxicity table for healthy volunteers, adapted to site laboratory normal reference ranges (see Appendix 2).

Immunological assessments. Sera were heat inactivated for 30 mins at $56^{\circ} \mathrm{C}$ prior to storage at $-20^{\circ} \mathrm{C}$ before assessment in immunological assays. Binding antibody concentrations induced by the vaccine in participant sera were assessed using an anti-S IgG ELISA in 96 well plates coated with the stabilised SARS-CoV-2 spike protein in the pre-fusion conformation. Background 
optical density (OD) 450nm readings from uncoated wells (blank wells) were subtracted from readings in test wells. Vaccine-induced seroconversion to anti-S IgG was considered to have occurred in those where there was an OD of above $0.2 \mathrm{~nm}$ in the ELISA. This threshold was set during optimisation of the ELISA using standards from National Institute for Biological Standards and Control (NIBSC), convalescent sera from individuals recovered from SARS-CoV-2 infection and sera taken prior to December 20I9. Baseline convalescent sera were available from 32 individuals enrolled into the wider COVACi trial with either a history of mild or moderate COVID or previously unknown, asymptomatic SARS-CoV-2 infection. A positive control of pooled plasma samples from NIBSC was included in each assay plate. When it became available (December 2020), the first WHO international standard antiSARS-CoV-2 immunoglobulin was added at a concentration of $2 \mathrm{BAU} / \mathrm{mL}$ as an additional control. It was determined that $2 \mathrm{BAU} / \mathrm{mL}$ was the equivalent of approximately $20,000 \mathrm{ng} / \mathrm{mL}$. For the immunoblots, Ioong of S protein was bound to a nitrocellulose plate and probed with sera from participants at baseline and week 6 . Blots were subsequently probed with Goat antihuman IgG - HRP Peroxidase to detect spike specific IgG bound to the membrane. The immunoblots were developed using Immobilon Crescendo Western HRP substrate and read using Chemiluminescence Imager CELVIN $^{\circledR}$. The integrated density of each immunoblot was calculated using the Fiji Image Processing Package. ${ }^{\mathrm{I2}}$ Data are reported as a continuous scale of the integrated density. A cut-off value for positivity of $\mathrm{I} \cdot 6 \log _{\text {го }}$ was determined from the distribution of values observed at baseline (Appendix 2, Figure 16). Further analysis was conducted to assess potential non-specific cross-reactivity in the immunoblot assay and to compare its sensitivity relative to ELISA (Appendix 2, Figures I7 \& I8).

SARS-CoV-2 neutralisation assays were conducted using pseudotyped (PSV) and wild type viruses at Imperial College London. Briefly for the PSV assay, pseudotyped SARS-CoV-2 lentiviruses were produced in HEK293T/I7 cells using a SARS-CoV-2 spike plasmid, HIV-I gag-pol plasmid and a firefly luciferase reporter. Participant sera were serially diluted and incubated with PSV viral supernatant for I hour. HEK-ACE2 cells were then co-incubated with the sera and PSV for up to 96 hours before measurement of the luciferase activity using the Steady-Glo Luciferase assay system (Promega, Madison, WI). IC50 neutralisation titres were calculated as the dilution at which relative luminescence was reduced by $50 \%$ compared with control. For the PSV assay, the First WHO International Standard for antiSARS-CoV-2 immunoglobulin was included as a positive control, which was determined to have an IC50 neutralisation titre of approximately i:3000.

Wild type SARS-CoV-2 neutralisation assays were conducted as previously described. ${ }^{5}$

\section{Outcomes}

The objectives were to compare the safety and immunogenicity of two injections with LNP-nCoVsaRNA given four weeks apart at six different dose levels. The safety outcome measures were solicited local injection site and systemic reactions that started within seven days of administration of the vaccine, and any of the following that occurred throughout the study period: unsolicited adverse reactions (ARs), serious adverse events (SAEs) and unsolicited AEs. These were reported through to four weeks post second vaccine dose. Immunogenicity was assessed by the titre of neutralising antibodies two and four weeks after the second injection, and the titre of IgG raised against the SARS-CoV-2 S glycoprotein two and four weeks after the first and the second injections.

\section{Statistical analysis}

Sample size was based on achieving adequate statistical power to detect significant differences in $\mathrm{IC}_{50}$ neutralisation titres $\mathrm{NT}_{50}$ across dose groups. Results from the dose escalation and dose evaluation components of the trial, and the low and high dose cohorts, were combined. This paper reports initial immunological findings at weeks 2, 4, 6 (vaccine $2+2$ weeks), and 8 (vaccine $2+4$ weeks). Two participants (allocated to 5.0 and $10.0 \mu \mathrm{g}$ ) were retrospectively identified as being anti-S IgG seropositive at enrolment and are excluded from all immunogenicity analyses (but retained in safety analyses). Binding and neutralisation antibody titres were analysed on a logarithmic scale and back transformed for presentation. Tests for trend across dose groups were performed by assessing the coefficient for $\log$ (dose) in logistic regression models (for seroconversion outcome) and normal linear regression models (for antibody titres among those who seroconverted). Safety outcomes across all doses and grades were compared using Fisher's exact test. The rate of reactions following the first and second vaccines were compared using the Stuart-Maxwell test for marginal homogeneity implemented in the Stata symmetry command. All analyses were carried out using Stata I6.0 (StataCorp, College Station, TX, USA).

\section{Role of the funding source}

The funders had no role in the study design, the collection, analysis and interpretation of data, writing of the report and the decision to submit for publication.

\section{Results}

\section{Recruitment and compliance}

A total of I92 participants were enrolled between ig June and 28 October 2020 (Figure I). Demographics of enrolled participants are described in Table I. The majority were male (II9/I92; 62\%) with median age 


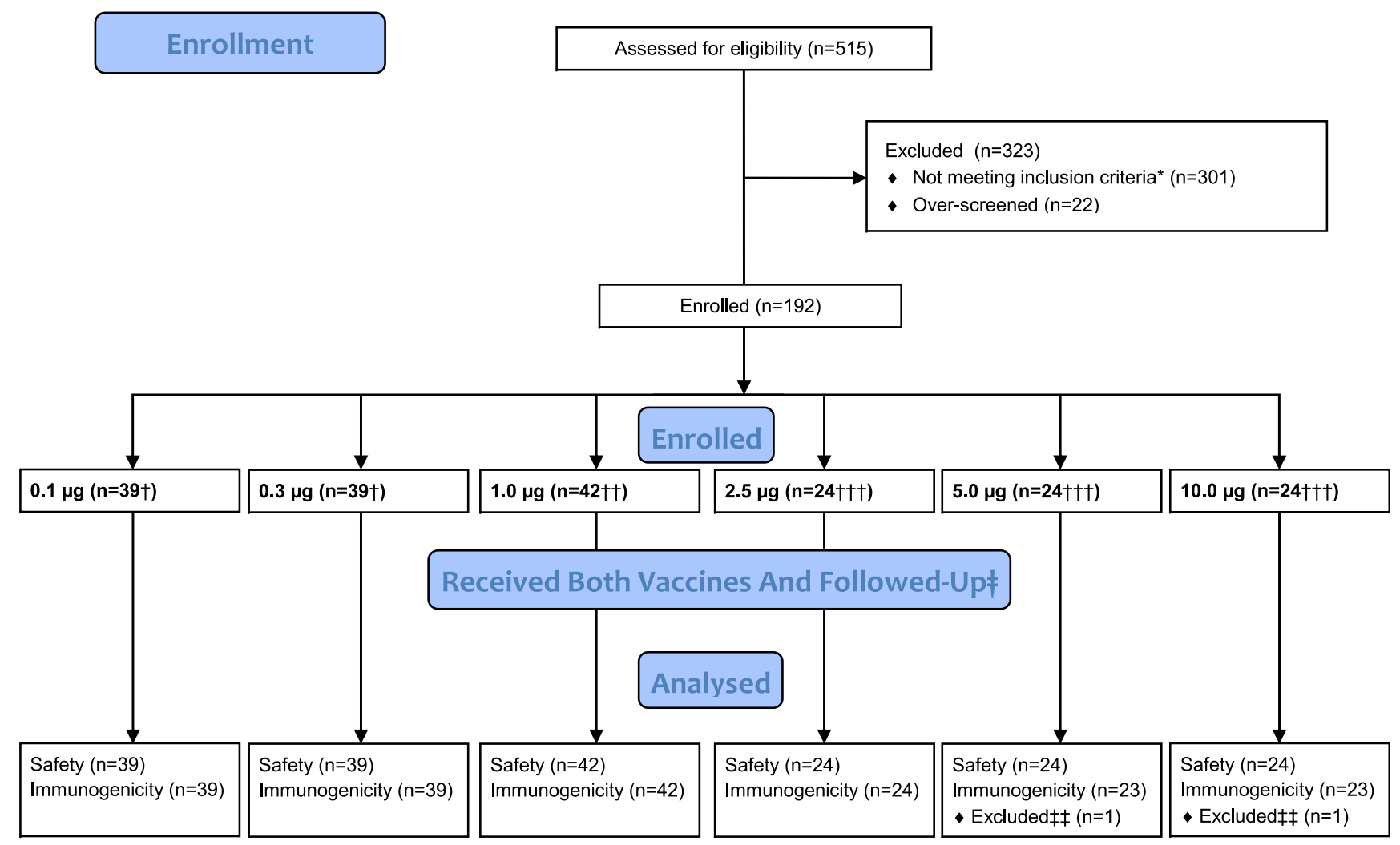

Figure 1. Consort diagram. Consort diagram demonstrating the eligibility assessment, enrolment, group allocation and follow-up of the $\mathrm{n}=192 \mathrm{participants} \mathrm{in} \mathrm{the} \mathrm{dose} \mathrm{escalation} \mathrm{and} \mathrm{rando-}$ mised dose evaluation components of the study. 
33 years and ethnicity representative of the UK population; $84(44 \%)$ were overweight or obese. All individuals enrolled received two injections; $5.7 \%$ (II/I92) were out of the window period for the second injection but are included in the analyses. Overall, 96\% (1479/1536) of visits were carried out in the window, including 97\% (I87/192) of vaccine $2+\mathrm{I} 4$ days and $96 \%$ (185/192) of vaccine $2+28$ days visits.

Reactogenicity. The proportion of participants reporting a systemic reaction increased significantly with dose reaching $100 \%$ of those receiving 5.0 or $10.0 \mu \mathrm{g}$ $(\mathrm{p}=0.000 \mathrm{I})$. Severity also increased with dose and four (I7\%) of 24 participants who received Io.o $\mu \mathrm{g}$ reported a grade 3 (severe) systemic reaction after the first or second vaccine (see Appendix 2, Table 5.4). Common reactions reported were fatigue (Io8/192; 56\%), headache (99; 52\%), myalgia (68; 35\%), arthralgia $(46 ; 24 \%)$, chills $(45 ; 23 \%)$ and nausea (34; I $8 \%$ ) (Figure $2 \mathrm{~B})$. Only one (I\%) of I44 participants in dose groups $0 . \mathrm{I}-2.5 \mu \mathrm{g}$ had a fever $\left(\geq 38^{\circ} \mathrm{C}\right)$, whereas eight $(17 \%)$ of 48 in the two highest dose groups recorded a fever, and in three participants in the two highest dose groups this was 39.0-40.0 $0^{\circ} \mathrm{C}$ (grade 3). No-one reported a severe local reaction, and 53/192 (28\%) reported no local reaction at all, but the frequency also increased significantly with dose ( $\mathrm{p}<0.000 \mathrm{I})$ and 47 (98\%) of 48 participants receiving 5.0 or $10.0 \mu \mathrm{g}$ reported a local reaction with one in three grading this as moderately severe. Overall, tenderness/discomfort and pain were reported by $133 /$ I9 $^{2}$ $(69 \%)$ and $73(38 \%)$ respectively; erythema $(5 ; 3 \%)$ and swelling (2; $1 \%)$ were uncommon (Figure 2A). Solicited reactions following the first and second vaccine were similar overall with no significant differences in maximum grade of systemic or local reactions $(\mathrm{p}=0.06$ Table 5.7 and $\mathrm{p}=0.52$ Table 5.9, Appendix 2 respectively). This was also the case when the test for marginal homogeneity was applied to each unique reaction other than headache, where higher grades of headache were more frequently observed following the second dose compared to the first ( $p=0.0$ I, Appendix 2, Table 5.8). Seven days after each vaccination, laboratory safety parameters remained largely within normal limits (Appendix 2 pI5-I6, Figures 3-I3).

Other adverse events. There were no SAEs considered related to the study intervention. Amongst the 137 (71\%) participants reporting 394 AEs, 25 had a moderately severe event, and six had a severe or worse event (Appendix 2, Tables 5.I0 and 5.II). Two of the six were hospital admissions (shigella infection, spinal fracture) and the other four were detected through grade 3 changes in routine laboratory parameters. Two participants had a transient grade 3 transaminitis associated with heavy exercise, a third had a grade 3 decrease in neutrophils considered to be due to untreated Graves' disease, and a fourth had asymptomatic hyperbilirubinaemia secondary to Gilbert's syndrome. Three participants had mild-to-moderate self-limiting AEs that merited a specialist opinion before proceeding with a second investigational vaccine or authorised COVID-I9 vaccine: a generalised rash following contact with food or topical agents; recurrent facial oedema determined to be stress-induced angioedema; and back pain accompanied by numbness and tingling in the extremities.

\section{Immunogenicity}

Binding antibody. Low rates of seroconversion to anti-S IgG were observed by ELISA in participants who received o.I or $0.3 \mu \mathrm{g}$, only $3 / 39(8 \%)$ and 10/39 (26\%) at week six (two weeks after the second vaccination), respectively (Table 2 ). The highest seroconversion rates occurred in the Io.o $\mu \mathrm{g}$ group, $8 / 23$ (35\%) four weeks after the first vaccination, increasing to $14 / 23$ (6I\%) two weeks after the second vaccination. Intermediate rates were observed in the I.०, 2.5 and $5.0 \mu \mathrm{g}$ groups. Among those who seroconverted, anti-S IgG titres appeared to be higher at six weeks in the $5.0 \mu \mathrm{g}$ group (GM=1023ng/ $\mathrm{mL})$ and $10.0 \mu \mathrm{g}$ group $(\mathrm{GM}=500 \mathrm{ng} / \mathrm{mL})$ compared with the lower dose groups. Most individual antibody concentrations ranged from approximately Ioong/mL to $2000 \mathrm{ng} / \mathrm{mL}$, broadly consistent with values derived from convalescent sera (Figure $3 \mathrm{~A}$ ).

Interestingly there were different patterns of response in the binding antibody (ELISA) according to time after the first and second injections. Thirty-two of I9O (I7\%) participants responded to a single prime dose and this included participants in every dose group from O.I-IO.o $\mu \mathrm{g}$ (Figure 4A). Among the 32 participants who did respond to the prime dose, five had no detectable response following the booster dose, I3 showed no or a marginal $(<0.5$ $\log _{\text {Io }}$ ) increase in antibody titre, while 13 had evidence of a boosting effect $\left(>0.5 \log _{\text {Iо }}\right.$ increase); one sample was not collected at week six. A boosting effect was more commonly observed in those receiving 5.0 or Io.o $\mathrm{gg}$ but was also observed in those receiving a lower dose.

Among the participants who showed no ELISA response four weeks after the prime dose, 37 (23\%) seroconverted by two weeks after the booster dose (Figure 4A). In those with a response at week six, most had a response at week eight (i.e. four weeks after the booster dose), but there were some whose response did not appear until week eight and others whose response was present at week six but lost at week eight (Figure ${ }_{4} \mathrm{~B}$ ). Again, these patterns of response were seen in all dose level groups.

\section{Neutralising antibody}

Neutralising antibody, as determined in a pseudovirus assay, is reported for samples obtained at weeks six and 
(a) Local
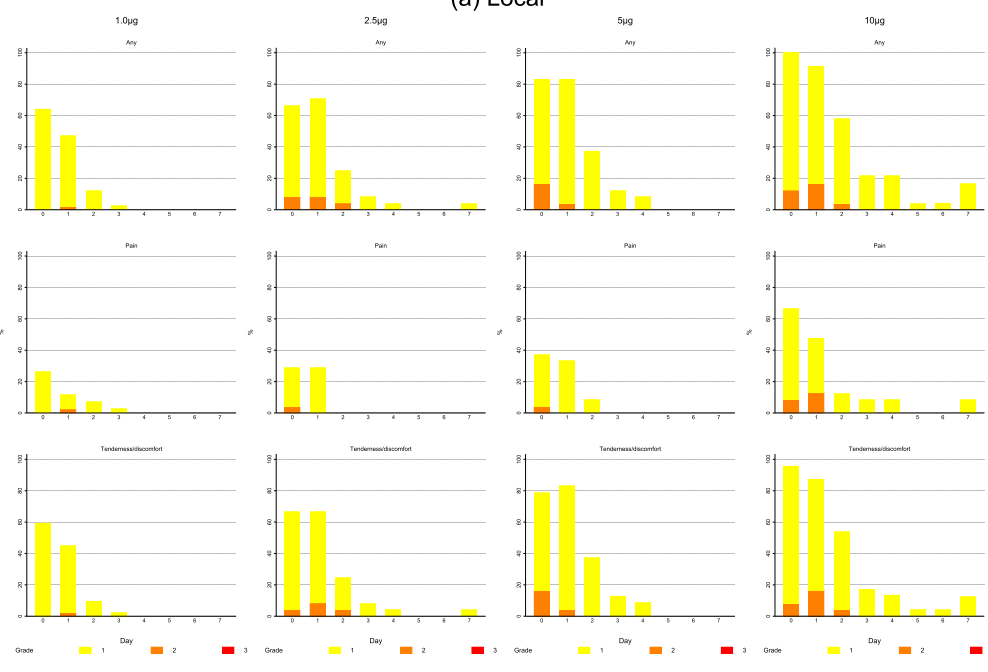

(b) Systemic
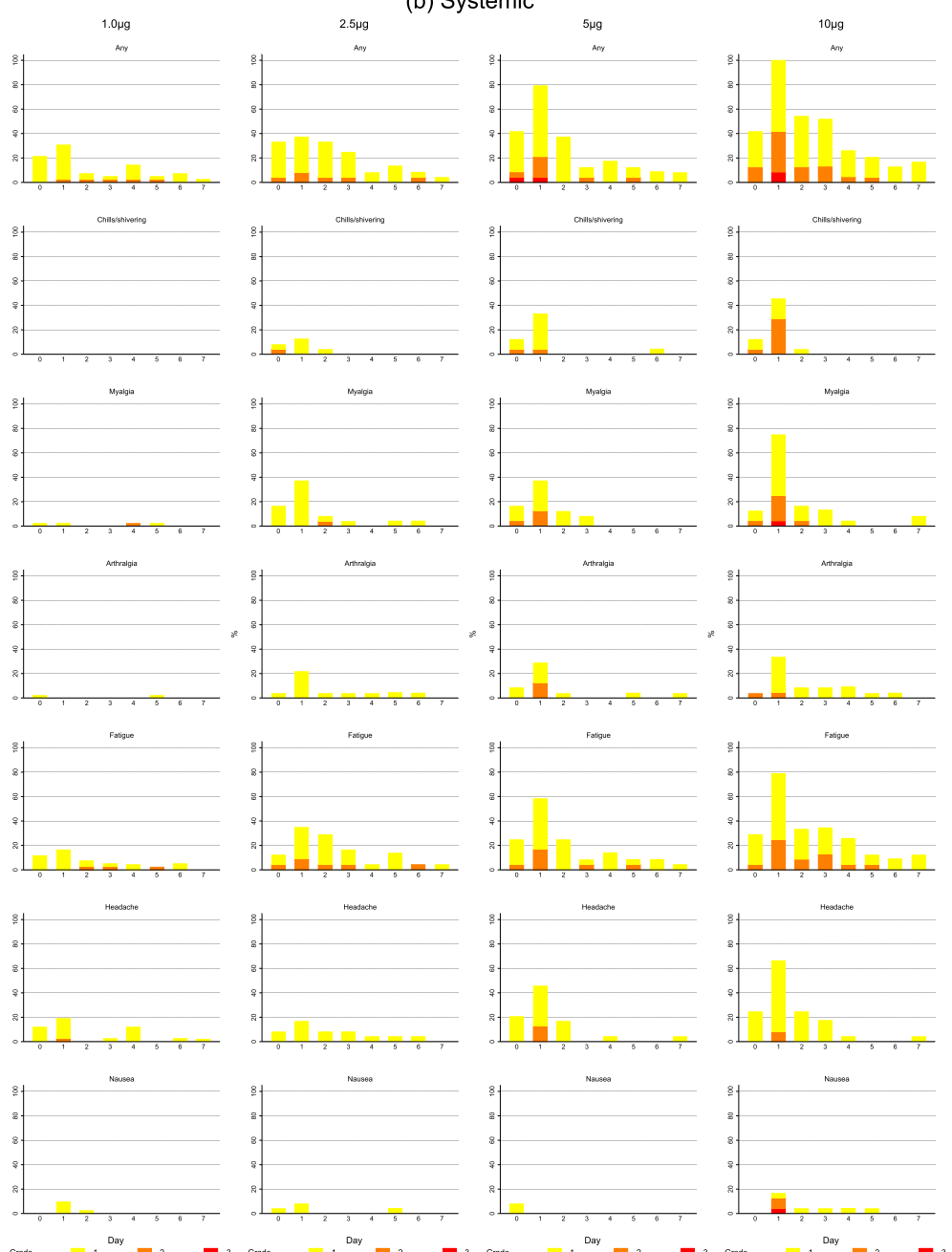

Figure 2. A. Solicited local injection site reactions that started within 7 days of administration of the vaccine with a frequency of at least $10 \%$. Reactions are shown after the first injection in those who received, in columns from left to right $1.0 \mu \mathrm{g}, 2.5 \mu \mathrm{g}, 5.0 \mu \mathrm{g}$, and 

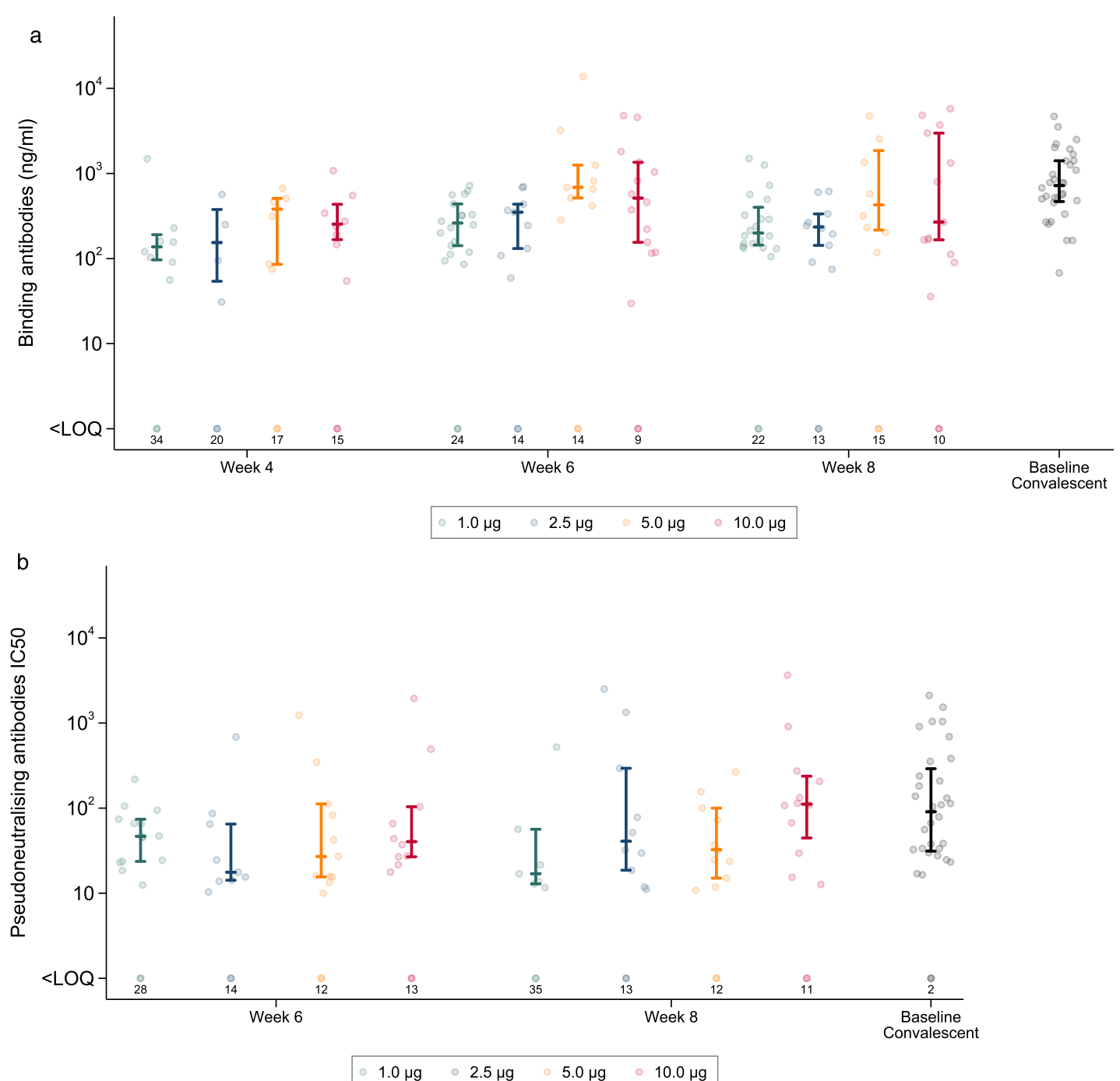

Figure 3. A. Anti-Spike (S) IgG $(\mathrm{ng} / \mathrm{mL})$ raised in sera from participants receiving two doses of LNP-saRNA. Responses are shown at 4 weeks, 6 weeks, and 8 weeks after enrolment in those who received $1.0 \mu \mathrm{g}$ (green dots), $2.5 \mu \mathrm{g}$ (blue dots), $5.0 \mu \mathrm{g}$ (orange dots), and $10.0 \mu \mathrm{g}$ (red dots). Responses from convalescent sera are shown as black dots - GM ( $95 \% \mathrm{Cl}): 718 \mathrm{ng} / \mathrm{mL}(518,996)$. Error bars detail the median and interquartile range amongst responders. Responses that did not meet criteria for a positive response are shown on the bottom row with numbers of participants $\angle \mathrm{LOQ}$ (limit of quantification). Figure 3B. Pseudoneutralising antibodies IC50 from participants receiving two doses of LNP-saRNA Responses are shown at 6 weeks and 8 weeks after enrolment in those who received $1.0 \mu \mathrm{g}$ (green dots), $2.5 \mu \mathrm{g}$ (blue dots), $5.0 \mu \mathrm{g}$ (orange dots), and $10.0 \mu \mathrm{g}$ (red dots). Responses from convalescent sera are shown as black dots - GM NT 50 (95\% Cl): 130 (74-229). Error bars detail the median and interquartile range amongst responders. Responses that did not meet criteria for a positive response are shown on the bottom row with numbers of participants < LOQ (limit of quantification).

$10.0 \mu \mathrm{g}$. The upper row shows reports of any solicited local injection site reaction, the middle row pain at the injection site and the lower row tenderness at the injection site on the day of vaccination and for 7 days afterwards. Grade of adverse event is represented by colour on the bar chart as grade 1 (mild) in yellow, grade 2 (moderate) in orange and grade 3 (severe) in red. Figure 2B. Solicited systemic reactions that started within 7 days of administration of the vaccine with a frequency of at least $10 \%$. Reactions are shown after the first injection in those who received in columns from left to right, $1.0 \mu \mathrm{g}, 2.5 \mu \mathrm{g}, 5.0 \mu \mathrm{g}$, and $10.0 \mu \mathrm{g}$. Rows show from the top any solicited systemic reaction, chills/shivering, myalgia, arthralgia, fatigue, headache and nausea. Grade of adverse event is represented by colour on the bar chart as grade 1 (mild) in yellow, grade 2 (moderate) in orange and grade 3 (severe) in red. 


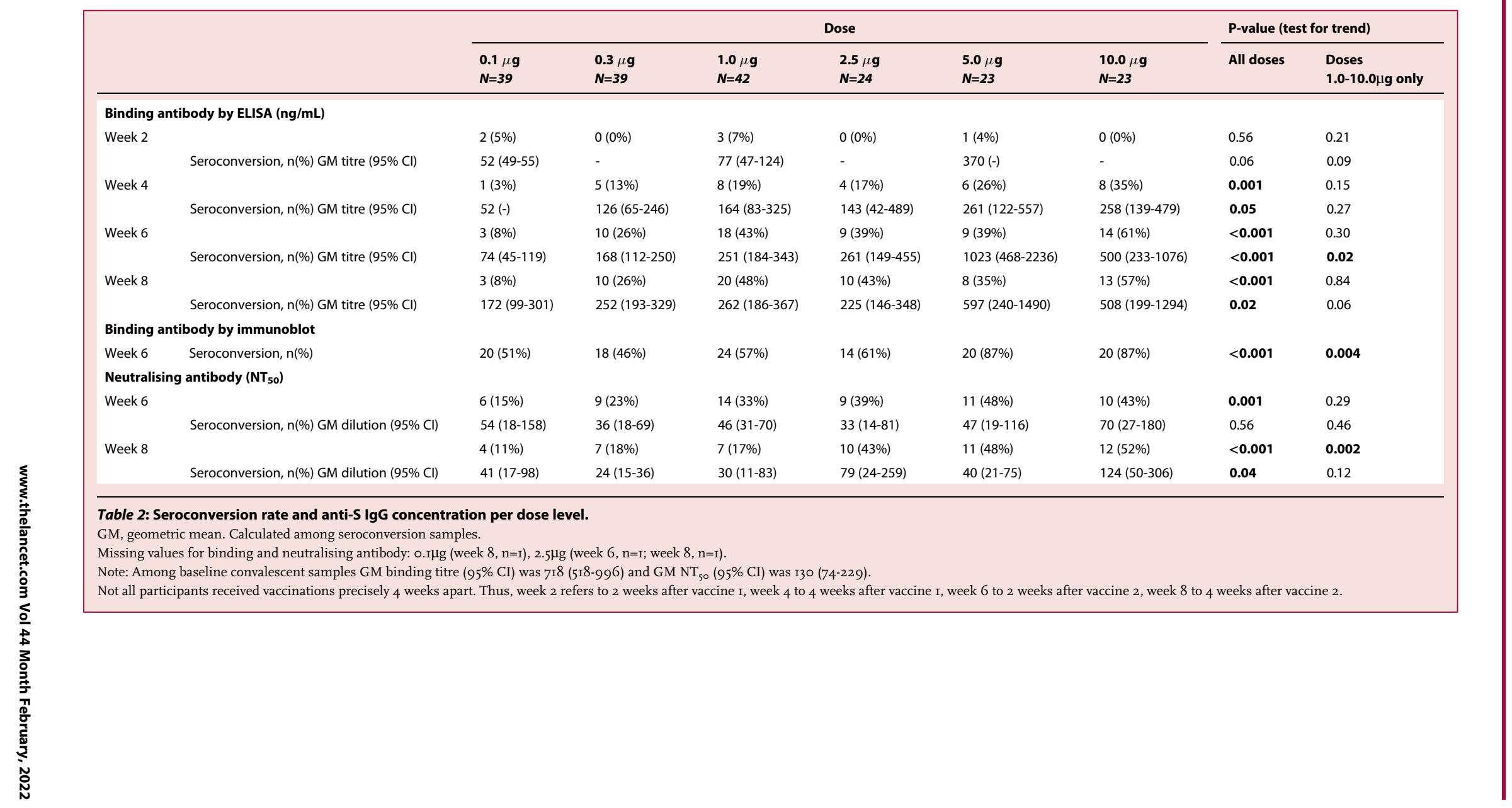



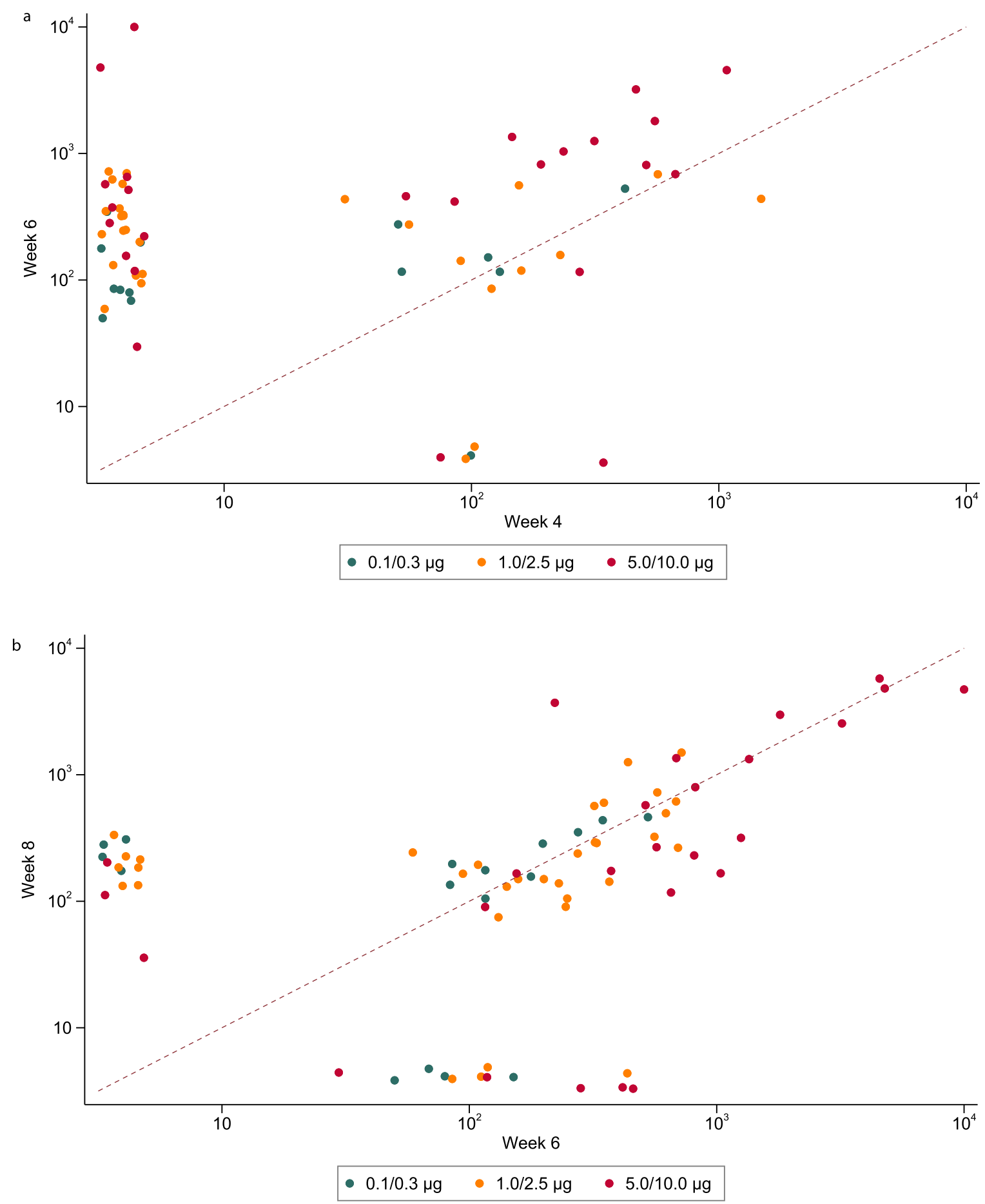

Figure 4. A. Anti-Spike (S) lgG ( $\mathrm{ng} / \mathrm{mL})$ raised in sera from participants at week 4 against week 6 after enrolment. Reponses from those who received 0.1 or $0.3 \mathrm{ug}$ (green dots), 1.0 or $2.5 \mathrm{ug}$ (orange dots) or 5.0 or $10.0 \mathrm{ug}$ are shown (red dots). The dashed line represents an equivalent binding response at both week 4 and week 6 , values above this line indicate an increased response (and a decreased response below this line) at week 6 in comparison to week 4. Figure $4 B$. Anti-Spike $(S) \mathrm{lgG}(\mathrm{ng} / \mathrm{mL})$ raised in sera from participants at week 6 against week 8 after enrolment. Reponses from those who received 0.1 or 0.3 ug (green dots), 1.0 or 2.5 ug (orange dots) or 5.0 or $10.0 \mathrm{ug}$ are shown (red dots). The dashed line represents an equivalent binding response at both week 6 and week 8 , values above this line indicate an increased response (and a decreased response below this line) at week 8 in comparison to week 6 . 
eight (Table 2). There was a statistically significant trend in the rate of detection of neutralising antibody across the dose groups, but with no clear difference between the $5.0 \mu \mathrm{g}$ group (II/23 (48\%) at week six) and the

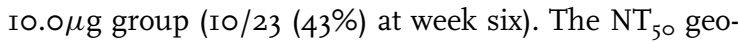
metric mean was higher in the Io $\mu \mathrm{g}$ group than in the $5.0 \mu \mathrm{g}$ group at both six weeks (70 versus 47 ) and eight weeks (I24 vs 40) but neither difference was statistically significant ( $\mathrm{p}=0.55$ and 0.06 respectively). $\mathrm{NT}_{50}$ values following the booster dose were somewhat lower than those observed on convalescent sera (Figure $3 \mathrm{~B}$ ). Neutralising antibodies were detected less often, and at lower titres, with the wild-type assay than with the pseudotyped assay (Appendix 2, Figure I4).

Sera from 25 individuals had neutralising activity measurable in the pseudovirus assay but no anti-S IgG binding at week six by ELISA (Appendix 2, Figure I5). To understand this discordance, as a post-hoc analysis, we screened sera at week $o$ and week 6 by immunoblot (Appendix 2, Figures I9). Overall seroconversion across all dose levels was $116 / 189$ (61\%), ranging from $20 / 39$ (51\%) and $18 / 39$ (46\%) for the lowest doses (O.I and $0.3 \mu \mathrm{hg}$ respectively) to $87 \%$ for $5.0(20 / 23)$ and Io.0 $\mu \mathrm{g}$ $(20 / 23)$ doses (Table I). A further eight individuals did not meet the criteria for seroconversion but demonstrated an appreciable change in signal intensity from baseline. We detected seroconversion in 9/25 (36\%) individuals where there was neutralisation but no binding antibody.

\section{Discussion}

There were no unexpected safety issues in this short period following two IM injections of LNP-nCoVsaRNA up to $10.0 \mu \mathrm{g}$ in this cohort of I92 adults aged I845 years. Two participants had adverse events that led to a delay in their second vaccines, which were administered with no recurrence. Reactogenicity was dose dependent, with the highest proportion of grade 3 reactions in those receiving Io. $\mu \mu \mathrm{g}$.

The safety profile of LNP-nCoVsaRNA appears similar to other mRNA COVID-I9 vaccines, where systemic and local reactions of grade 2 and above were common, particularly in younger adults. ${ }^{\text {I3-I5 }}$ We saw no evidence of clinically significant potentiation after the second dose, beyond a slight increase in grade 2 headaches. No allergic events were considered related to the saRNA vaccine ${ }^{16}$ although this may reflect exclusion of subjects with a significant allergy history. Doses of I.० $\mu \mathrm{g}$ and below were associated with very low levels of reactogenicity. Seroconversion by ELISA was sensitive to dose level, with maximum rates (I4/23; 6I\%) at I0.0 $\mu \mathrm{g}$. However, none of the groups reached roo\% seroconversion with a range of $39 \%(9 / 23 ; 2.5 \mu \mathrm{g}$ and $5.0 \mu \mathrm{g})$ to 6I $\%$ (I4/23; I0.0 $\mu \mathrm{g}$ ) for the I.O-IO.০ $\mu$ g groups. The highest titre of anti-S IgG antibody was in those receiving $5.0 \mu \mathrm{g}$ and was not further augmented in those receiving double this dose. Where seroconversion by ELISA occurred, anti-S IgG concentration was in the same range as convalescent sera, neutralisation IC50 was slightly lower. The discordance between neutralisation and seroconversion by ELISA triggered assessment of seroconversion by immunoblot assay. This revealed a higher seroconversion rate of $\mathrm{iI} 6 / 189$ (6ı\%) across all doses, ranging from $18 / 39(46 \%)$ for $0.3 \mu$ to $20 / 23$ $(87 \%)$ for the two highest doses (5.0 and Io.o $\mu \mathrm{g})$. Differences between seroconversion by ELISA and immunoblot reflect differences in sensitivity (see Appendix 2, Figure I8) between the two assays. This may reflect differences in the presentation of binding epitopes on immobilisation of the spike glycoprotein to ELISA plates versus nitrocellulose membrane. ${ }^{\mathrm{I7}}$ However, I6/ 59 participants positive by pseudovirus neutralizing assay were negative by both ELISA and immunoblot. Although observed by others, ${ }^{\mathrm{I}}$ the reason for such discordance is unclear but may reflect exposure of epitopes on pseudotyped virions not accessible or represented by the purified recombinant protein.

Interestingly, for on-going development, immune responses were observed at low dose levels (I.o $\mu \mathrm{g}$ ) and even at ultralow dose levels (0.I $\mu \mathrm{g}, 20 / 39$ (5I\%) by immunoblot). Nevertheless, the proportion of individuals generating neutralizing antibodies (I4/42 (33\%) and $6 / 39$ ( $15 \%$ ) respectively) would not be sufficient for an effective vaccine. We present here an interim report of data obtained, the study is on-going, and participants will be followed for I year. Data on cellular responses, epitope mapping and persistence of antibody will be reported elsewhere.

In this saRNA vaccine design, only non-structural proteins are derived from VEEV preventing potentially infectious genetic reversion, reactogenicity from immune response to structural alphavirus proteins, or off-target immunity. Pre-clinical studies of our LNPnCoVsaRNA vaccine demonstrated high levels of neutralising anti-S IgG even when given at $0.0 \mathrm{I} \mu \mathrm{g}$, with a dose response across a three-log range. ${ }^{5}$ Due to constraints with vaccine preparation, the volume of administration for the o.I $\mu \mathrm{g}$ dose level was $0.2 \mathrm{~mL}$. It is conceivable that this may have affected this very low dose. However, immunogenicity was also low at $0.3 \mu \mathrm{g}$, and the most plausible explanation is that below I. $\circ \mu \mathrm{g}$ there was insufficient RNA payload within target cells leading to suboptimal antigen expression.

At I.o $\mu \mathrm{g}$ and above, the dose response by ELISA was more complex and hints at a potential mechanism for the level of non-responders. Whilst increasing dose increased the reactogenicity, concentrations of anti-S IgG were not linearly affected, likely indicating persistently low antigen expression. It is possible that recognition of both lipid nanoparticle and/or saRNA exposure by endosomal and cytoplasmic pathogen-associated molecular pattern receptors (e.g. TLRs, RIG-I \& MDA5) may restrict saRNA amplification and antigen 
expression. ${ }^{19,20}$ Differences in threshold for triggering such pathways at an individual level may have influenced initiation of self-amplification and determined whether antigen expression levels were sufficient for seroconversion by ELISA and/or immunoblot. As the threshold for innate sensing is influenced by the basal state of immune activation ${ }^{2 \mathrm{I}, 22}$ and given the different patterns of response to the first or second injection irrespective of dose, it is conceivable that innate activation from the first immunisation may have impacted on the second. Indeed, the replication kinetics of saRNA, with antigen expression occurring for up to $2 \mathrm{I}$ days, ${ }^{\text {IO,II }}$ suggests a four-week interval between doses may not be optimal where a longer interval may be required to return to a more quiescent state.

Pre-clinical models have been used to test an increasing breadth of candidate saRNA vaccines based on: VEEV; a combination of VEEV plus Sindbis virus; classical swine fever virus; tick-borne encephalitis virus; or Semliki Forest virus (reviewed in). ${ }^{23}$ Our saRNA backbone was derived from the virulent Trinidad donkey strain of VEEV rather than its attenuated vaccine derivative, strain TC-83. ${ }^{24}$ The latter includes a G3A mutation in the 5' untranslated region which increases sensitivity to IFN $-\alpha / \beta$ and alters the secondary structure of this region. ${ }^{24}$ Understanding the impact of different 5' UTRs on variability of immune response to saRNA and the attenuated VEEV TC83 strain used for vaccination ${ }^{25}$ could provide further insight to the development of this platform. Indeed, clinically, very little is currently known about how different saRNA designs perform. Current knowledge is likely to be further informed by two other candidate saRNA vaccines now in clinical trials, against rabies (NCTo4062669) and COVID-I9 (NCTo4480957).

The two mRNA COVID-I9 vaccines (Moderna and Pfizer/BioNTech) now in widespread clinical use ${ }^{26,27}$ performed better than our LNP-saRNA, demonstrating Io०\% seroconversion.

Both mRNA vaccines use a similar, if not identical 2P-S presentation of the $\mathrm{S}$ glycoprotein as used in this study, formulated in LNPs, the Pfizer vaccine using identical lipids. ${ }^{27}$ These mRNA vaccines, incorporate the modified nucleobase methyl-pseudouridine to minimise innate recognition of the mRNA using higher doses (100 $\mu \mathrm{g}$ and $30 \mu \mathrm{g}$ for Moderna and Pfizer respectively) than those in this study. However, the compatibility of modified bases with saRNA has not been established and these would be rapidly lost on amplification. Furthermore, induction of innate restriction pathways triggered within hours may have a greater impact on saRNA than mRNA given differences in kinetics of expression. ${ }^{\text {IO,II }}$ Importantly the observed immunogenicity in humans was inferior to that observed in mice. This likely reflects differences in innate restriction of exogenous RNA sensing. ${ }^{28-3 \mathrm{I}}$ Understanding species specific differences with respect to innate restriction of saRNA expression may prove key to unlocking the true potential of this technology for humans and development of more predictive animal models. It is probable that these issues can be resolved through further refinement of saRNA vaccine design such as the inclusion of encoded modulators of human pattern recognition receptors (now undergoing clinical evaluation), ${ }^{32}$ use of alternative UTRs and a wider range of potential modifications. ${ }^{33}$

This is the first human study to report on the response to LNP encapsulated saRNA based on an alphavirus replicon in humans. These data provide preliminary data into this novel RNA platform technology. Nevertheless, responses with this construct were insufficient to meet the target for translation in Phase 3 trials and further work is needed to refine the platform. However, global demand for COVID-I9 vaccines will remain high in the coming decade, given the emergence of lethal SARS-CoV-2 escapevariants and expected requirement for booster vaccination..$^{33}$ If modified to improve antibody titres, encapsulated saRNA could provide potential advantages for vaccine development including scalability, tolerability, and flexibility in antigen design, to meet requirements for the ongoing response to the COVID-I9 pandemic.

\section{Contributors}

COVACI was designed by DTD, SMc, KMP and RS. KMP was the Chief Investigator and SMc provided clinical oversight. HMC coordinated the laboratory data collection and oversight. SM was the Qualified Person and TC oversaw the pharmacy procedures. Raw data provided by the Imperial College London Section of Immunology of Infection laboratory were accessed by the MRC Clinical Trials Unit COVAC I study statisticians (AS, HB and DTD) for analysis. AS and HB conducted the analyses with advice from HMC, DTD, SMc, KMP and RS. MB, CC, SF, HH, VL, KP and AW were site Principal Investigators. SMc, KMP, RS and JNW were study members of the Trial Steering Committee; HB, AS and HMC attended as observers. KMP and RS wrote the first draft of the paper and HB, HMC, DTD, SMc and AS contributed. All other authors contributed to the implementation of the study, analysis and data collection. All authors critically reviewed and approved the final version of the manuscript and took the decision to submit the manuscript for publication.

\section{Declaration of interests}

P.F.M. and R.J.S. are co-inventors on a patent application covering this SARS-CoV-2 saRNA vaccine. All the other authors have nothing to report.

\section{Data sharing}

Data will be made available when the trial is complete, upon requests directed to the corresponding author and after approval of a proposal. 


\section{Acknowledgments}

We wish to acknowledge the COVACi participants who took part in this first in human vaccine trial, the dedication of the clinic, laboratory and administrative teams, and the oversight that the governance committees provided as the study evolved, including Jonathan Weber who represented the Sponsor, Sue Marlow as Qualified Person, the members who represented the Patients and Public (PPI) and the members of the Independent Data Monitoring Committee established to oversee the planned efficacy trial. Manufacture studies were additionally supported by the NIHR Imperial Biomedical Research Centre (BRC) and the MRC Imperial confidence in Concept. Preclinical toxicology was funded by Department of Health and Social Care using UK Aid funding, managed by the Engineering and Physical Sciences Research Council (EPSRC, grant number: EP/ RoI3764/I). Infrastructure support was provided by the NIHR Imperial Biomedical Research Centre and the NIHR Imperial Clinical Research Facility. We also acknowledge Fondation Dormeur, Vaduz and a gift from the James B. Pendleton Charitable Trust for providing funds to purchase equipment used in these studies. DTD and SMc were supported by a Medical Research Council grant, MC_UU_I2023/23 \& MC_UU_00004/04. KMP was supported by the NIHR Imperial BRC. We would like to acknowledge also members of the COVACi Governance (Independent members): Trial Steering Committee: Robert Lechler (Chair), Janet Darbyshire (Vice Chair), Jeff Almond, Ben Cromarty (PPI), Stuart Elborn;

Independent Data Monitoring Committee (for planned COVAC2): Peter Smith (Chair), George Griffin, Phil Minor, Moira Whyte; PPI representative on the Trial Management Group: Pro Chatzikyriakou.

\section{Funding}

This study was co-funded by grants and gifts from the Medical Research Council UKRI (MC_PC_I9076), and the National Institute Health Research/Vaccine Task Force, Partners of Citadel and Citadel Securities, Sir Joseph Hotung Charitable Settlement, Jon Moulton Charity Trust, Pierre Andurand, Restore the Earth.

\section{Supplementary materials}

Supplementary material associated with this article can be found in the online version at doi:Io.IoI $6 / j$. eclinm.202I.IOI262.

\section{References}

I Fontanet A, Autran B, Lina B, Kieny MP, Karim SSA, Sridhar D. SARS-CoV-2 variants and ending the COVID-I9 pandemic. Lancet 2021;397:952-4.

2 Teijaro JR, Farber DL. COVID-I9 vaccines: modes of immune activation and future challenges. Nat Rev Immunol 2021;21:I95-7.
3 Bernstein DI, Reap EA, Katen K, et al. Randomized, doubleblind, Phase I trial of an alphavirus replicon vaccine for cytomegalovirus in CMV seronegative adult volunteers. Vaccine 2009;28:484-93.

4 Wecker M, Gilbert P, Russell N, et al. Phase I safety and immunogenicity evaluations of an alphavirus replicon HIV-I subtype C gag vaccine in healthy HIV-I-uninfected adults. Clin Vaccine Immunol 20I2;I9:I65I-60.

5 McKay PF, Hu K, Blakney AK, et al. Self-amplifying RNA SARS$\mathrm{CoV}-2$ lipid nanoparticle vaccine candidate induces high neutralizing antibody titers in mice. Nat Commun 2020;II:3523.

6 Sandbrink JB, Shattock RJ. RNA Vaccines: A Suitable Platform for Tackling Emerging Pandemics? Front Immunol 2020;1I:608460.

7 Kis Z, Shattock R, Shah N, Kontoravdi C. Emerging Technologies for Low-Cost, Rapid Vaccine Manufacture. Biotechnol J 2019;I4: eI800376.

8 Rupp JC, Sokoloski KJ, Gebhart NN, Hardy RW. Alphavirus RNA synthesis and non-structural protein functions. J Gen Virol 20I5;96:2483-500.

9 Wrapp D, Wang N, Corbett KS, et al. Cryo-EM structure of the 20I9-nCoV spike in the prefusion conformation. Science 2020;367:1260-3.

io Huysmans H, Zhong Z, De Temmerman J, et al. Expression Kinetics and Innate Immune Response after Electroporation and LNPMediated Delivery of a Self-Amplifying mRNA in the Skin. Mol Ther Nucleic Acids 20I9;17:867-78.

II Blakney AK, McKay PF, Ibarzo Yus B, Hunter JE, Dex EA, Shattock RJ. The Skin You Are In: Design-of-Experiments Optimization of Lipid Nanoparticle Self-Amplifying RNA Formulations in Human Skin Explants. ACS Nano 20I9;13:5920-30.

I2 Schindelin J, Arganda-Carreras I, Frise E, et al. Fiji: an open-source platform for biological-image analysis. Nature methods 2012;9:67682. $20 \mathrm{I} 2$.

I3 Baden LR, El Sahly HM, Essink B, et al. Efficacy and Safety of the mRNA-I273 SARS-CoV-2 Vaccine. N Engl J Med 2021;384:403-I6.

I4 Polack FP, Thomas SJ, Kitchin N, et al. Safety and Efficacy of the BNTi6zb2 mRNA Covid-I9 Vaccine. $N$ Engl $J$ Med 2020;383:2603-I5

I5 Kremsner Peter, Mann Philipp, Bosch Jacobus, et al. Phase I Assessment of the Safety and Immunogenicity of an mRNA- Lipid Nanoparticle Vaccine Candidate Against SARS-CoV-2 in Human Volunteers. medRxiv 2020. .II.09.2022855I.

I6 Banerji A, Wickner PG, Saff R, et al. mRNA Vaccines to Prevent COVID-I9 Disease and Reported Allergic Reactions: Current Evidence and Suggested Approach. J Allergy Clin Immunol Pract 2021;9:I423-37.

I7 Butler JE, Navarro P, Sun J. Adsorption-induced antigenic changes and their significance in ELISA and immunological disorders. Immunol Invest I997;26:39-54.

I8 Kremsner P, Mann P, Bosch J, et al. Phase I Assessment of the Safety and Immunogenicity of an mRNA- Lipid Nanoparticle Vaccine Candidate Against SARS-CoV-2 in Human Volunteers. medRxiv 2020. https://doi.org/IO.IIOI/2020.II.09.2022855I.

I9 Miyake K, Shibata T, Ohto U, et al. Mechanisms controlling nucleic acid-sensing Toll-like receptors. Int Immunol 2018;30:43-5I.

20 Rehwinkel J, Gack MU. RIG-I-like receptors: their regulation and roles in RNA sensing. Nat Rev Immunol 2020;20:537-5I.

2I Martin SF. Adaptation in the innate immune system and heterologous innate immunity. Cell Mol Life Sci 20I4;7I:4II5-30.

22 Baden LR, El Sahly HM, Essink B, et al. Efficacy and Safety of the mRNA-I273 SARS-CoV-2 Vaccine. N Engl J Med 202I;384:403-I6.

23 Bloom K, van den Berg F, Arbuthnot P. Self-amplifying RNA vaccines for infectious diseases. Gene Ther 2021;28:II7-29.

24 Kulasegaran-Shylini R, Thiviyanathan V, Gorenstein DG, Frolov I. The 5'UT'R-specific mutation in VEEV TC-83 genome has a strong effect on RNA replication and subgenomic RNA synthesis, but not on translation of the encoded proteins. Virology 2009;387:2II-2I.

25 Pittman PR, Makuch RR, Mangiafico JA, Cannon TL, Gibbs PH, Peters CJ. Long-term duration of detectable neutralizing antibodies after administration of live-attenuated VEE vaccine and following booster vaccination with inactivated VEE vaccine. Vaccine I99 6; I4:337-43.

26 Polack FP, Thomas SJ, Kitchin N, et al. Safety and Efficacy of the BNTi6zb2 mRNA Covid-I9 Vaccine. N Engl J Med 2020;383:2603-I5.

27 Buschmann MD, Carrasco MJ, Alishetty S, Paige M, Alameh MG, Weissman D. Nanomaterial Delivery Systems for mRNA Vaccines. Vaccines 2021;9:65. 
28 Heil F, Hemmi H, Hochrein H, et al. Species-specific recognition of single-stranded RNA via toll-like receptor 7 and 8. Science 2004:303:I526-0.

29 Hagai T, Chen X, Miragaia RJ, et al. Gene expression variability across cells and species shapes innate immunity. Nature 2018;563:197-202

30 Zschaler J, Schlorke D, Arnhold J. Differences in innate immune response between man and mouse. Crit Rev Immunol 2014;34:433-54.
3I Mestas J, Hughes CC. Of mice and not men: differences between mouse and human immunology. I Immunol 2004;I72:273I-8.

32 Blakney AK, McKay PF, Bouton CR, Hu K, Samnuan K, Shattock RJ. Innate Inhibiting Proteins Enhance Expression and Immunogenicity of Self-Amplifying RNA. Mol Ther 202I;29:II74-85.

33 Excler JL, Saville M, Berkley S, Kim JH. Vaccine development for emerging infectious diseases. Nat Med 202I;27:59I-600. 